\title{
Facet-Dependent Interactions of Islet Amyloid Polypeptide with Gold Nanoparticles: Implications for Fibril Formation and Peptide-Induced Lipid Membrane Disruption
}

\author{
Shih-Ting Wang, ${ }^{\dagger, \ddagger}$ Yiyang Lin, ${ }^{\dagger,+}$ Nevena Todorova, ${ }^{\S}$ Yingqi Xu, ${ }^{\|}$Manuel Mazo, ${ }^{\dagger,}$ Subinoy Rana, ${ }^{\dagger, \ddagger}$
} Vincent Leonardo, ${ }^{\dagger, \dagger}$ Nadav Amdursky, ${ }^{\dagger, \ddagger}$ Christopher D. Spicer, ${ }^{\dagger, \dagger}$ Bruce D. Alexander, ${ }^{\perp}$

Alison A. Edwards, " Steve J. Matthews," Irene Yarovsky, ${ }^{\S}$ and Molly M. Stevens ${ }^{* \dagger, \ddagger \subset}$

${ }^{\dagger}$ Department of Materials, Imperial College London, London SW7 2AZ, U.K.

${ }^{\ddagger}$ Department of Bioengineering and Institute of Biomedical Engineering, Imperial College London, London SW7 2AZ, U.K.

${ }^{\S}$ School of Engineering, RMIT University, GPO Box 2476, Melbourne, Victoria 3001, Australia

"Department of Life Sciences, Imperial College London, London SW7 2AZ, U.K.

${ }^{\perp}$ Department of Pharmaceutical, Chemical and Environmental Science, University of Greenwich, Central Avenue, Chatham, Kent ME4 4TB, U.K.

${ }^{\#}$ Medway School of Pharmacy, Universities of Kent and Greenwich at Medway, Central Avenue, Chatham, Kent ME4 4TB, U.K.

Supporting Information

\begin{abstract}
A comprehensive understanding of the mechanisms of interaction between proteins or peptides and nanomaterials is crucial for the development of nanomaterialbased diagnostics and therapeutics. In this work, we systematically explored the interactions between citrate-capped gold nanoparticles (AuNPs) and islet amyloid polypeptide (IAPP), a 37-amino acid peptide hormone co-secreted with insulin from the pancreatic islet. We utilized diffusion-ordered spectroscopy, isothermal titration calorimetry, localized surface plasmon resonance spectroscopy, gel electrophoresis, atomic

Facet dependent Au-IAPP affinity

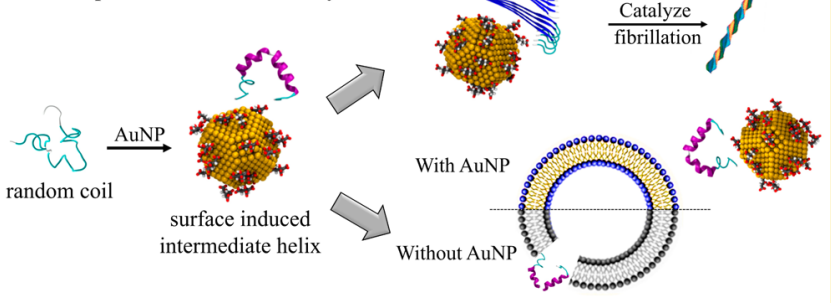
force microscopy, transmission electron microscopy (TEM), and molecular dynamics (MD) simulations to systematically elucidate the underlying mechanism of the IAPP-AuNP interactions. Because of the presence of a metal-binding sequence motif in the hydrophilic peptide domain, IAPP strongly interacts with the Au surface in both the monomeric and fibrillar states. Circular dichroism showed that AuNPs triggered the IAPP conformational transition from random coil to ordered structures $(\alpha-$ helix and $\beta$-sheet), and TEM imaging suggested the acceleration of IAPP fibrillation in the presence of AuNPs. MD simulations revealed that the IAPP-AuNP interactions were initiated by the N-terminal domain (IAPP residues $1-19$ ), which subsequently induced a facet-dependent conformational change in IAPP. On a $\mathrm{Au}(111)$ surface, IAPP was unfolded and adsorbed directly onto the $\mathrm{Au}$ surface, while for the $\mathrm{Au}(100)$ surface, it interacted predominantly with the citrate adlayer and retained some helical conformation. The observed affinity of AuNPs for IAPP was further applied to reduce the level of peptide-induced lipid membrane disruption.
\end{abstract}

\section{INTRODUCTION}

Islet amyloid polypeptide (IAPP) is an amyloidogenic peptide involved in the pathogenesis of type II diabetes (T2D), an epidemic disease worldwide that is associated with the selfassembly and deposition of fibrillar plaques in the pancreatic islets. $^{1-7}$ Physiologically, IAPP is a 37 -amino acid peptide hormone that is co-secreted with insulin and involved in glycemic regulation. ${ }^{6}$ In a manner similar to those of other amyloid peptides, IAPP contains a hydrophobic C-terminal domain that governs fibrillation kinetics and a hydrophilic domain at the $\mathrm{N}$-terminal domain that assists with structural stability. The driving force for IAPP self-assembly into amyloid fibrils is ascribed to hydrophobic interactions and hydrogen bonding in the core amyloidogenic region (SNNFGAILSS,
IAPP 20-29) at the C-terminal domain. ${ }^{8}$ A similar fibrillation mechanism has also been found in many other amyloid peptides and/or proteins such as those related to Alzheimer's disease and Parkinson's disease. ${ }^{9}$ Under normal conditions, IAPP is inherently disordered, with self-assembly being inhibited by insulin and other chaperones. ${ }^{7,10}$ However, overproduction of $\beta$-sheet rich IAPP fibrils at the pancreatic islet has been found in $90 \%$ of T2D individuals, in which the deposition of fibrillar plaques and associations with the cell membranes are related to the apoptosis and loss of pancreatic

Received: September 28, 2016

Revised: January 25, 2017

Published: February 13, 2017 
Scheme 1. (a) Schematic View of the Sequence of IAPP, in Which the N-Terminal Domain Binds to the Cell Membrane and the Amyloidogenic Core (residues 20-29) Dictates Self-Assembly of Unstructured Monomers into $\beta$-Sheet Rich Amyloid Fibrils, and (b) a Proposed Schematic Displaying the Two Routes of IAPP-AuNP Interaction ${ }^{a}$

a)

Membrane binding region Amyloidogenic core

IAPP KCNTATCATQRLANFLVHSSNNFGAILSSTNVGSNTY

b)

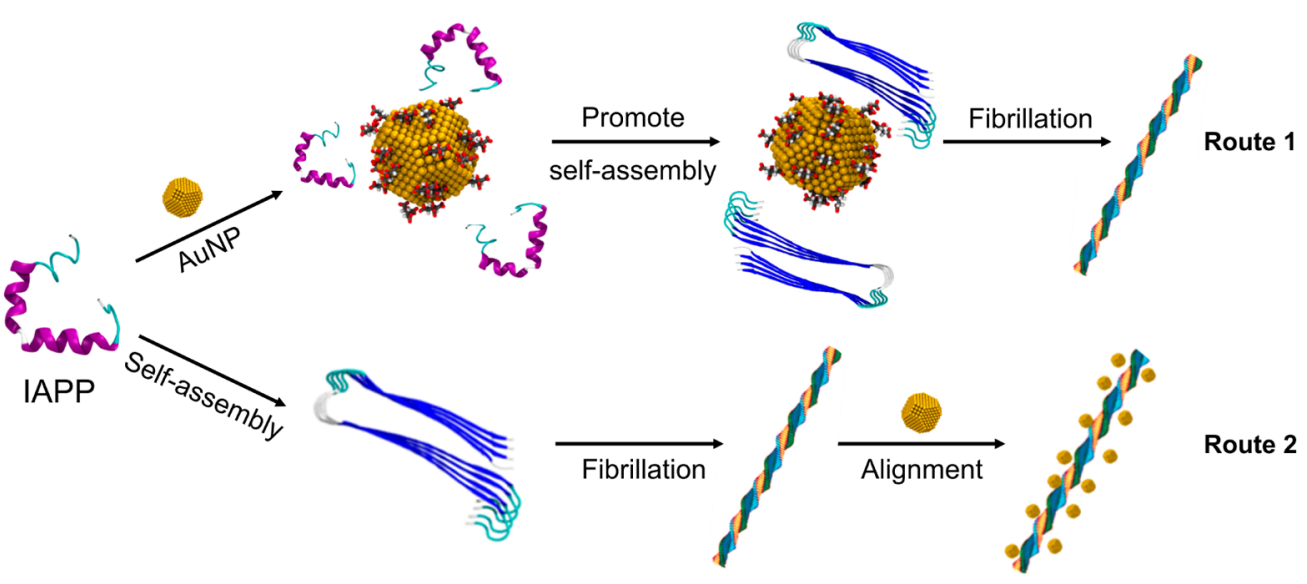

${ }^{a}$ Route 1, acceleration of IAPP nucleation and fibril growth during the self-assembly process upon interaction with the AuNP surface. Route 2, preformed IAPP fibrils inducing the alignment of AuNPs. Note that routes 1 and 2 can occur in parallel once IAPP is dissolved in buffer.

$\beta$-cells. ${ }^{2,7}$ Much effort has been made to design amyloid inhibitors to suppress IAPP fibrillation. ${ }^{11-16}$

Amyloid peptides in their oligomeric ${ }^{9,17-21}$ or fibrillar ${ }^{22-25}$ forms have been reported to be involved in disease pathologies and cytotoxicity through interactions with cellular membranes. During T2D, IAPP has been proposed to play an important role in ion channel formation and nonspecific disruption of lipid membranes, which disturbs homeostasis and results in $\beta$ cell apoptosis. ${ }^{26-29}$ Because of the presence of lysine (K1), arginine (R11), and histidine ( $\mathrm{H} 18)$ residues, the $\mathrm{N}$-terminal domain (residues 1-19) carries a net positive charge under physiological conditions. Previous research using phosphatidylglycerol (PG)- and phosphatidylserine (PS)-based lipid models has shown that the positively charged $\mathrm{N}$-terminal domain can initiate binding of IAPP to anionic lipids, causing IAPP to adopt an $\alpha$-helical conformation on the negatively charged surface. ${ }^{30-33}$ Similar conformational changes have been shown to be a key driving force for the intermolecular recognition necessary for the formation of $\beta$-sheet rich amyloid. ${ }^{17,21,34-36} \mathrm{~A}$ high concentration of localized amyloid peptides can integrate on lipid bilayers and alter the amyloid formation pathway. ${ }^{21,31}$

Over the past decade, nanomaterials have found broad applications in the field of diagnostics and therapeutics because of their stability, large surface area, controllable morphology, and unique physical properties. ${ }^{37,38}$ Understanding the fundamental molecular interactions at the bio-nano interface is essential to extending the current applications of nanomaterials. Indeed, the association of biological molecules such as proteins, peptides, and nucleic acids with nanomaterials is a complex process, because multiple interactions can be cooperatively involved in binding. The presence of nanoparticle (NP) surfaces can induce changes in the secondary structure of proteins, resulting in an increased diversity and complexity of protein structures at the interface, which makes it more complicated to understand the protein-NP interaction at the molecular level. ${ }^{39-42}$ Of particular interest is the interaction between amyloid peptides and NP surfaces, with NPs of different sizes and surface compositions having been reported to govern the kinetic processes of peptide fibrillation, and its related cytotoxicity. ${ }^{43-47}$ NP-induced inhibition or acceleration of amyloid fibrillation has been reported. These processes are influenced by multiple factors, including the size, shape, surface charge, and hydrophobicity of NPs. ${ }^{43,48-53}$

In this work, the interaction between IAPP and citratecapped gold NPs (AuNPs) is studied by combining synergistic experiments and all-atom simulations. We anticipate that understanding the IAPP-AuNP interaction and its effects on IAPP self-assembly will help reveal the mechanisms of nanomaterial-amyloid peptide interactions at the molecular level and may open interesting directions for the discovery of new agents that interfere with the fibrillation process.

\section{RESULTS AND DISCUSSION}

Observation of the Strong Affinity of IAPP for AuNPs. As shown in Scheme 1a, the peptide sequence of IAPP consists of a hydrophilic N-terminal domain (residues 1-19) that can interact with the cell membrane and a hydrophobic amyloidogenic core (residues 20-29) at the C-terminal domain that promotes peptide fibrillation. Interestingly, residues that can bind to the Au surface, including lysine (K1), cysteine (C2 and $\mathrm{C} 7$ ), asparagine (N3 and N14), arginine (R11), and histidine (H18), are mostly located within the N-terminal domain of IAPP. ${ }^{54,55}$ These multiple binding sites may initiate strong binding of IAPP to the AuNP surface, which is expected to cause IAPP deformation and modulate the nucleationgrowth pathway in parallel with the peptide self-assembly process (route 1 , Scheme $1 \mathrm{~b}$ ). ${ }^{53}$ Moreover, because the Aubinding sequence of IAPP is located at the $\mathrm{N}$-terminal domain, which is exposed on the fibril surface, preformed fibrillar nanostructures would also interact with AuNPs and induce particle alignment (route 2).

We used proton diffusion-ordered nuclear magnetic resonance spectroscopy $\left({ }^{1} \mathrm{H}\right.$ DOSY-NMR or DOSY) to examine the affinity of IAPP for AuNPs. DOSY is a powerful tool for investigating molecular interactions by measuring the diffusion coefficients of solute molecules, ${ }^{56}$ which allowed us to 


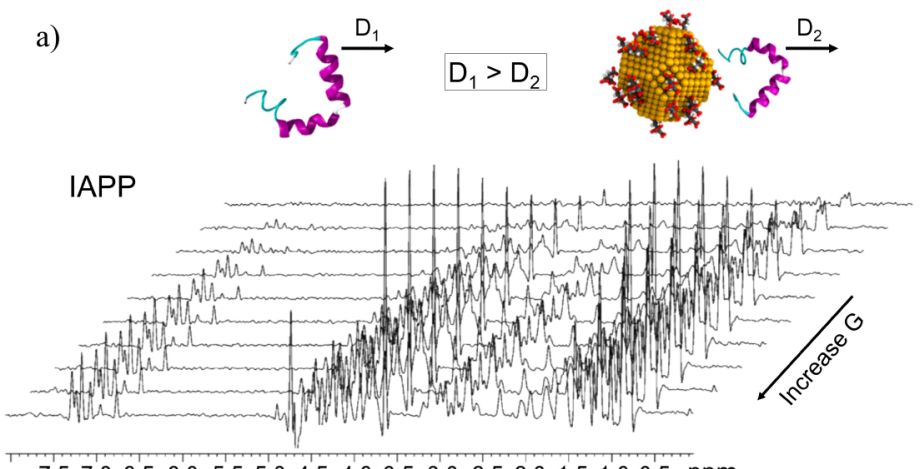

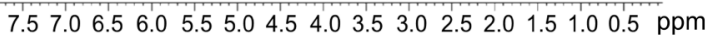
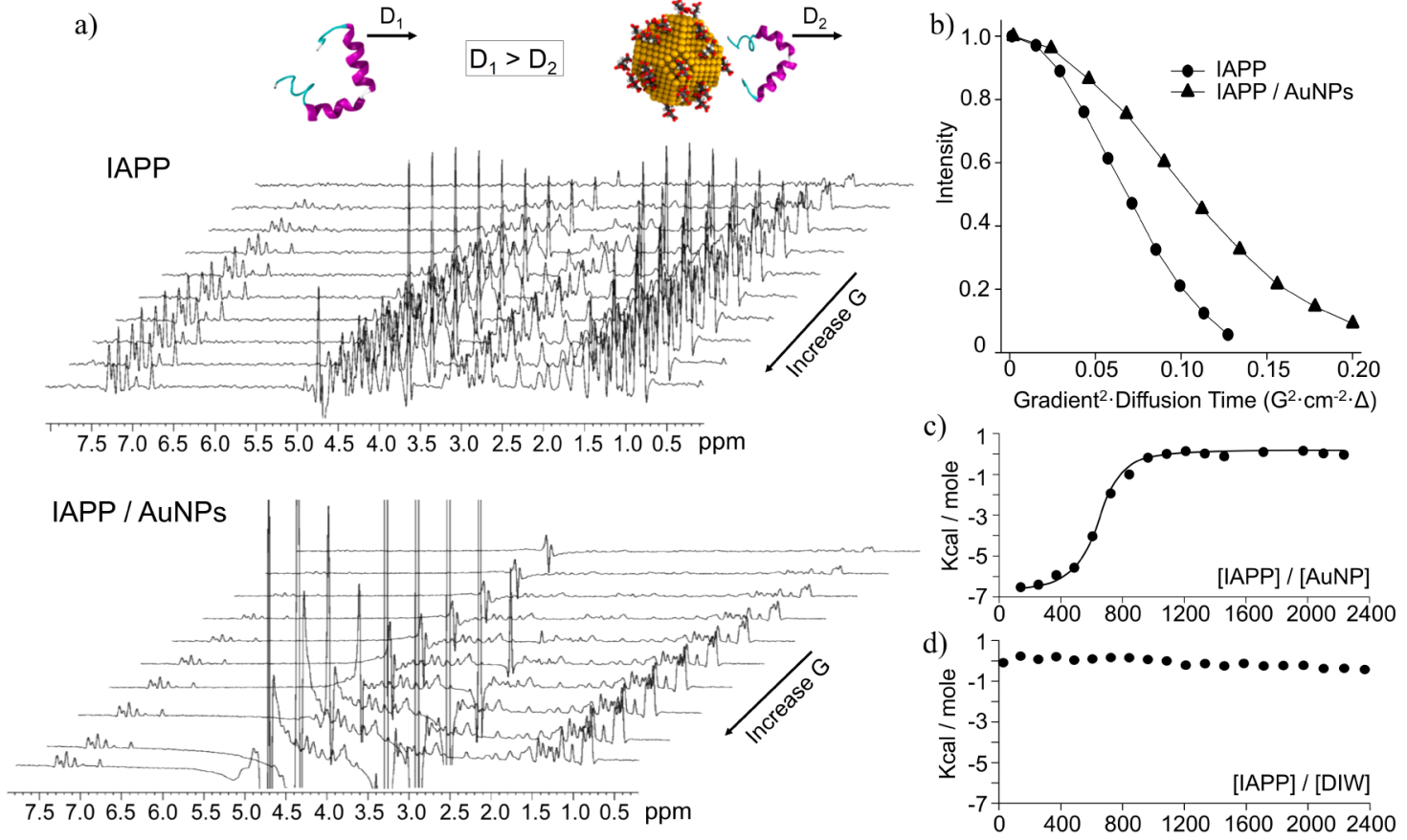

Figure 1. Investigation of the interactions between IAPP and citrate-capped AuNPs. (a) Schematic view of the reduced mobility of IAPP caused by the binding of AuNPs. This was demonstrated by acquiring the DOSY spectra at different pulsed field magnetic strengths: (top) IAPP and (bottom) IAPP/AuNP ( $2 \mathrm{~nm})$. (b) Via a plot of the integrated NMR intensity vs magnetic field gradient, the diffusion coefficient $(D)$ was calculated, showing the reduced molecular mobility of IAPP in the presence of $2 \mathrm{~nm}$ AuNPs. (c) Curve fitting of the ITC raw data by a single set of binding sites, showing the interaction of IAPP with $5 \mathrm{~nm}$ AuNPs was a spontaneous exothermic process. The result was subtracted from the (d) control data (titration of IAPP into water).

demonstrate the binding of IAPP to $2 \mathrm{~nm}$ AuNPs. As shown in Figure 1, a series of spin echo spectra were measured with different pulsed field gradient strengths, and signal decays were analyzed to extract a set of diffusion coefficients $(D)$. The DOSY spectra of IAPP were acquired using optimized parameters for a maximal decay of $\sim 95 \%$ to be observed. The diffusion coefficient was calculated using the relative intensities in the region of $0.70-1.25 \mathrm{ppm}$, which in general corresponded to the $\alpha$ - and $\beta$-protons of the IAPP peptide (Figure 1 and Figure S1). Compared to free IAPP, which had a diffusion coefficient of $3.8 \times 10^{-10} \mathrm{~m}^{2} \mathrm{~s}^{-1}$ in solution, the diffusivity of IAPP in the presence of $2 \mathrm{~nm}$ AuNPs was reduced to $1.5 \times 10^{-10} \mathrm{~m}^{2} \mathrm{~s}^{-1}$, indicating that the binding of IAPP to AuNPs reduces the extent of Brownian motion because of the larger construct. To determine which part of IAPP was involved in binding to AuNPs, a series of one-dimensional CarrPurcell-Meiboom-Gill (CPMG) spectra were recorded for IAPP in solution and AuNP-bound IAPP (data not shown). While $T_{2}$ remains almost the same for most IAPP peaks, it was observed that a number of peaks assigned to the $\mathrm{N}$-terminal residues of IAPP $(\mathrm{C} 2 / \mathrm{N} 3, \mathrm{~T} 4, \mathrm{~A} 5 \text {, and T6 })^{57,58}$ exhibited a significant increase in spin-spin relaxation (e.g., $T_{2}$ relaxation times for T6 decreased from 23 to $19 \mathrm{~ms}$ ) in the presence of 2 nm AuNPs. In addition, many other peaks were less attenuated than those corresponding to $\mathrm{N}$-terminal residues, indicating the stronger binding to the latter. The affinity of IAPP for the AuNP surface was demonstrated by the mobility of AuNPs ( 5 $\mathrm{nm}$ ) in a peptide solution during agarose gel electrophoresis. As shown in Figure S2, it is clear that the reduced mobility of AuNPs (displayed as red bands) was observed at a peptide concentration as low as $0.1 \mu \mathrm{M}$. This effect can be explained by the partial charge neutralization of AuNPs by IAPP, because IAPP carries two positive charges at $\mathrm{pH} 7.5$, from lysine and arginine, at the N-terminal domain, while citrate-capped AuNPs are negatively charged (Table S1). When IAPP binds, the surface charge density of AuNPs is reduced and migration thereby blocked.

The IAPP-AuNP interactions and peptide-triggered AuNP aggregation were demonstrated by the peak shift of the plasmonic spectra. According to the DLVO theory, colloidal particles in aqueous solution are stabilized by electrostatic repulsion and steric effects. ${ }^{59}$ Here, the surface charge shielding of AuNPs upon the adsorption of IAPP resulted in immediate AuNP aggregation as observed by UV-vis spectroscopy (Figure S3a-c) with a red-shift of local surface plasmon resonance (LSPR) peaks being noted. A large red-shift was found for larger NPs $(20 \mathrm{~nm})$, for which an IAPP concentration as low as $0.31 \mu \mathrm{M}$ was able to trigger an $\sim 80 \mathrm{~nm}$ peak shift. A further increase in IAPP concentration led to a decrease in the peak shift (Figure S3d), possibly because the increased level of deposition of IAPP on the AuNP surface provides a steric effect to reduce the interparticle distance.

To assess the thermodynamic properties of IAPP-AuNP interactions, isothermal titration calorimetry (ITC) was performed to determine the binding affinity, enthalpy changes upon binding, and binding stoichiometry. As shown in Figure S4, the titration of IAPP into the AuNP $(5 \mathrm{~nm})$ solution showed that the binding of IAPP to AuNP surfaces was a spontaneous exothermic process $(\Delta H=-6.6 \pm 0.11 \mathrm{kcal} /$ $\mathrm{mol}$ ). The sigmoidal titration curve (Figure 1c) was generated by subtracting the result from the control data [titration of IAPP into water (Figure 1d)] to remove the cooperative effect of inter-IAPP interactions and the desolvation/dilution effect during peptide titration. Actually, the control data show no noticeable heat change, indicating that the strong exothermic response was mainly attributed to the IAPP-AuNP interaction. 

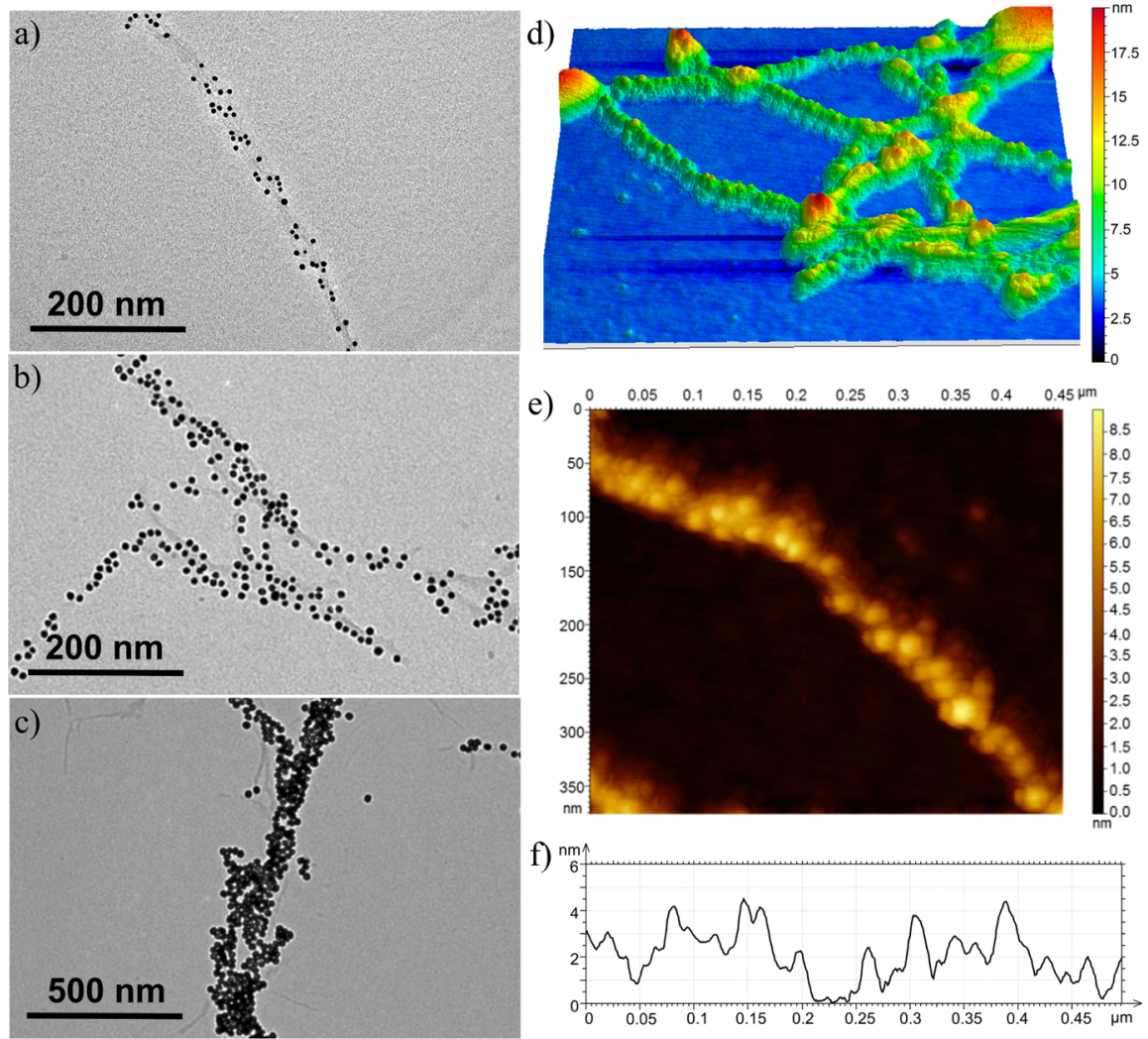

Figure 2. $(\mathrm{a}-\mathrm{c})$ TEM images showing the assembly of citrate-capped Au particles on preformed IAPP fibrils: $5 \mathrm{~nm}$ AuNPs, $10 \mathrm{~nm}$ AuNPs, and 20 $\mathrm{nm}$ AuNPs, respectively. ( $\mathrm{d}$ and e) AFM topographic images in tapping mode and (f) the corresponding height profile obtained from panel e showing the binding of $5 \mathrm{~nm}$ AuNPs on the IAPP fibril.

This ITC titration curve fitted well with a single-site binding process with a nonlinear least-squares regression, allowing the average number of binding sites $(N)$, dissociation constants $\left(K_{\mathrm{D}}\right)$, and entropy $(\Delta S)$ and enthalpy $(\Delta H)$ changes to be determined. A $K_{\mathrm{D}}$ of $\sim 9.18 \times 10^{6} \mathrm{M}^{-1}$ was observed, which was close to the binding limit for the complexation of the protein or peptide with AuNPs. ${ }^{60,61}$ The high estimated number of binding sites $(N \sim 600)$ could suggest a multilayered binding of IAPP to the AuNPs. In fact, the presence of a multilayer construct has been reported previously for the binding of $\beta_{2} \mathrm{~m}$ amyloid peptides on $N$-isopropylacrylamide/ $N$-tert-butylacrylamide NPs, which was attributed to the conformational restriction of the peptide on the particle surface. ${ }^{43}$ However, the result was not conclusive because AuNP agglomeration and peptide conformation changes could occur during the titration process. In a control experiment, the binding affinity of the IAPP amyloidogenic region (residues 20-29) for AuNPs was found to be much weaker, with the heat change acquired by ITC being significantly smaller (Figure S5). Moreover, the addition of freshly dissolved IAPP 20-29 peptide or its fibrillar form did not cause any LSPR peak shift from the $20 \mathrm{~nm}$ AuNP solution (Figure S6). These results suggest that the N-terminal domain of IAPP plays a crucial role in the IAPP-AuNP interaction, as confirmed by theoretical simulations discussed below.

It is known that the hydrophobic core of IAPP is embedded in its fibrillar structure, while the hydrophilic $\mathrm{N}$-terminal domain is located at the outer surface. ${ }^{62}$ Therefore, it is expected that Au-binding sites would be exposed to the aqueous environment in the fibrillar form. As a result, preformed IAPP fibrils (Scheme 1) can interact with citrate- capped AuNPs of different sizes and induce NP assembly on the fibrils, as displayed by TEM (Figure $2 a-c$ ) and atomic force microscopy (AFM) (Figure 2d-f). Using AFM, a packing of 5 $\mathrm{nm}$ AuNPs on the peptide fibril was observed to have an average height of 4-6 $\mathrm{nm}$ and a particle-particle spacing of $\sim 34 \mathrm{~nm}$ (Figure 2f). Unlike the freshly dissolved IAPP, the IAPP fibrils did not cause large plasmonic peak shifts of the AuNPs, presumably because the immobilization of AuNPs on fibrils prevented NP aggregation (Figure S7). ${ }^{63}$ In situ nucleation of AuNPs in the presence of IAPP fibrils was performed to show the strong interactions between AuNPs and peptide fibrils, although it remains unclear whether the formation of $\mathrm{Au}$-fibril hybrid structures was due to a templating effect or a post-synthesis adsorption. As shown in Figure S8a, AuNPs around $16 \pm 3 \mathrm{~nm}$ in size were preferentially formed on the IAPP fibril after reducing $\mathrm{Au}$ ions in 4-(2-hydroxyethyl)-1-piperazineethanesulfonic acid (HEPES) buffer. A dark field scanning transmission electron microscopy (DF-STEM) image (Figure S8b) and the corresponding elemental analysis by energy-dispersive X-ray spectroscopy (EDS) (Figure S8c) confirmed Au formation on the IAPP fibrils.

To gain insight into the interaction mechanisms and conformational changes of IAPP during the adsorption on citrate-capped AuNPs, molecular dynamics (MD) simulations were performed on the monomeric and preformed fibrillar structures of IAPP (Figure S9). The simulations indicated that IAPP monomers exhibited a facet-dependent conformational preference upon binding to citrate-capped $\mathrm{Au}(100)$ and $\mathrm{Au}(111)$ surfaces (Figure 3a). Starting from the helical IAPP conformation that was characteristic for a surface-adsorbed 
a)

- Unfolded $=$ Helical $=\beta$-strand $=$ Dynamic $(\mathrm{U} / \mathrm{H})$
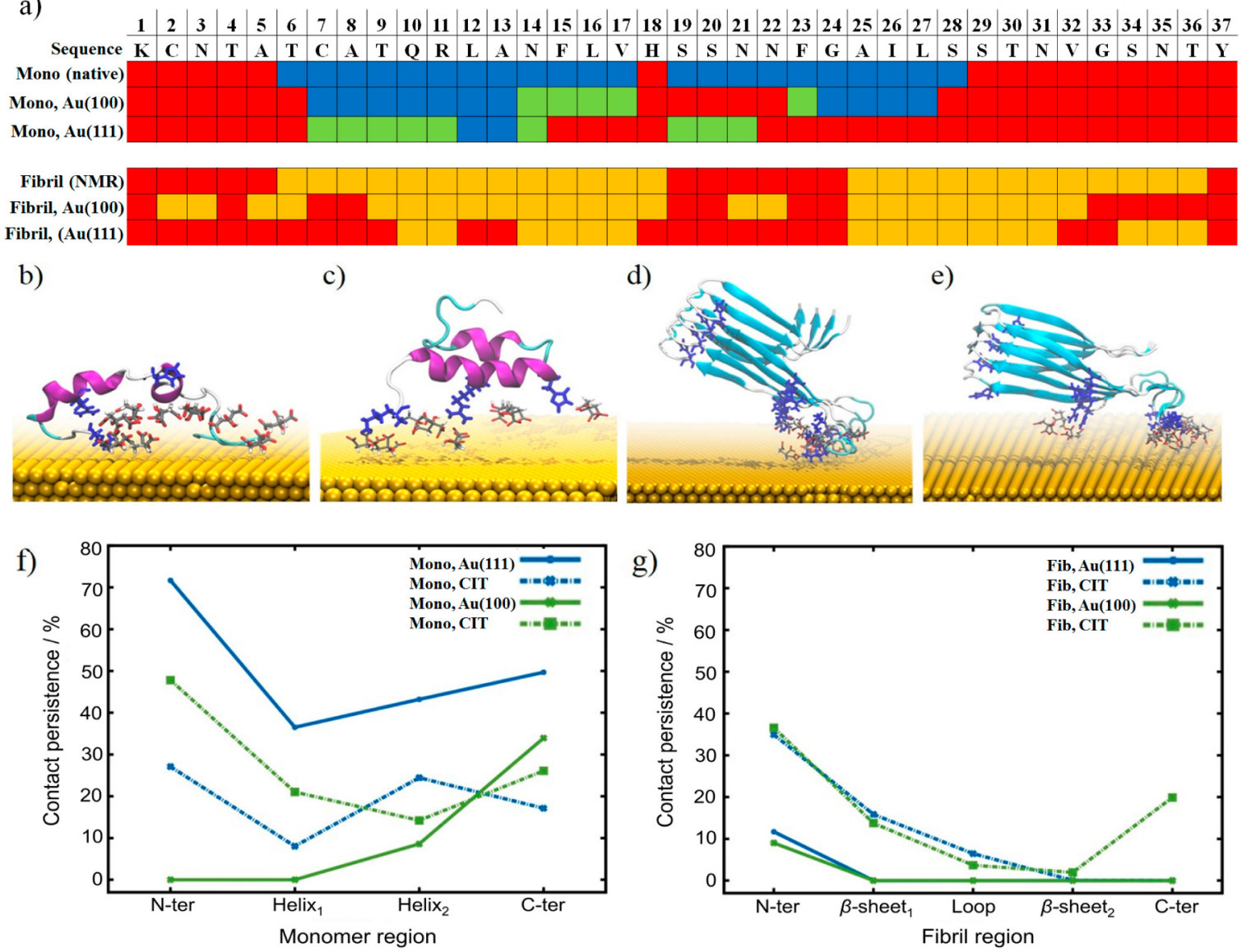

Figure 3. MD results showing (a) the conformational preference (per residue) of IAPP monomers and fibrils to adsorb on $\mathrm{Au}(100)$ and $\mathrm{Au}(111)$ surfaces. Representative structures of IAPP interacting with each surface: (b) monomer-Au(100), (c) monomer-Au(111), (d) fibril-Au(100), and (e) fibril- $\mathrm{Au}(111)$. The interactions between charged residues are colored blue, and the citrate adlayer within $3 \AA$ of IAPP is shown. IAPP is colored on the basis of its secondary structure: purple for helix, light blue for $\beta$-sheet, white for coil, and cyan for turn. Water molecules were omitted for the sake of clarity. Contact persistence of specific regions of IAPP: (f) monomers and (g) fibrils with the Au(111) (blue) and Au(100) (green) surfaces and the citrate adlayer (shown as dashed lines for each surface).

state and independent of the starting peptide orientation and $\mathrm{Au}$ facets, all simulations revealed that the initial contact between IAPP and the Au surface occurred via the N-terminal domain. This was likely due to the strong electrostatic attraction between the positively charged $\mathrm{K} 1$ residue and the negative citrate layer. In the presence of the $\mathrm{Au}(100)$ surface, IAPP largely retained its two helical regions, with some unfolding seen in the loop region at H18. The peptide interacted mostly with the citrate layer, where the helical conformation was stabilized by interactions between cationic $\mathrm{K} 1$ and $\mathrm{H} 18$ residues and the anionic citrate layer (Figure $3 \mathrm{~b}$ ). Interestingly, the second helix within the amyloidogenic region (residues 20-29) remained only partially folded and solventexposed, which could allow further conformational transition and subsequent fibril growth. This behavior is consistent with that seen for the binding of IAPP to anionic lipids, where the formation of an $\alpha$-helix between residues 5 and 22 has been observed. $^{30-33}$ Such a structure has also been postulated to be the key conformational motif that facilitates intermolecular recognition and amyloidogenesis. ${ }^{17,21,34-36}$ On the $\mathrm{Au}(111)$ surface, IAPP monomers interacted more strongly with the $\mathrm{Au}$ surface, driven by specific contacts between the amino acid residues and exposed $\mathrm{Au}$ atoms (Figure $3 \mathrm{f}$ ). This contributed to the partial unfolding of helix 1 (residues 6-17) and complete unfolding of the second helix (residues 20-29) (Figure 3a), which is involved in the self-assembly and fibril formation of IAPP. ${ }^{8}$ Thus, the unfolding observed for the hydrophobic Cterminal peptide domain in the presence of the $\mathrm{Au}(111)$ surface would be favorable for inducing interpeptide interactions and could promote fibril formation. On the other hand, the fibrillar form of IAPP retained its conformation over the entire simulation despite the exposed $\mathrm{Au}$ surface facet, suggesting that the fibril was stable in the presence of $\mathrm{Au}$. This favorable binding of IAPP fibrils to Au surfaces is in agreement with the TEM and AFM results shown in Figure 2. As for the IAPP monomers, the adsorption of IAPP fibrils on Au surfaces is likely dominated by the $\mathrm{N}$-terminal domain. However, the binding relies on electrostatic interactions with the citrate adlayer rather than direct contact with the Au surface (Figure 3d,e).

The role of water in the mechanisms of binding of IAPP to the Au surfaces was also investigated (Figure S10). Simulations showed a higher density of water on the $\mathrm{Au}(100)$ surface than on the $\mathrm{Au}(111)$ surface. This effect is due to the square arrangement of $\mathrm{Au}$ atoms on $\mathrm{Au}(100)$ surfaces that can facilitate structuring of water and marks a difference with the inplane quasi-hexagonal atomic arrangement of the $\mathrm{Au}(111)$ surface. The more compact adsorbed water layer resulted in 
a)

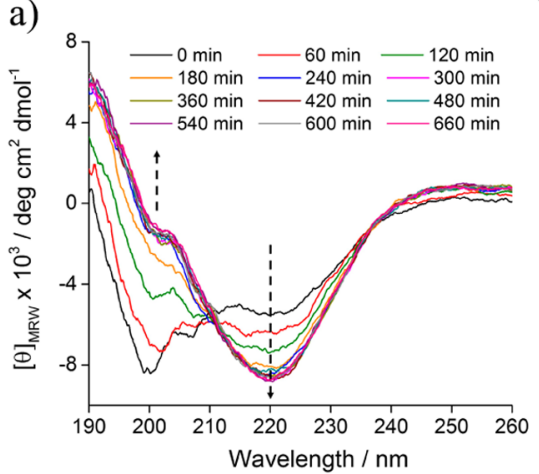

d)

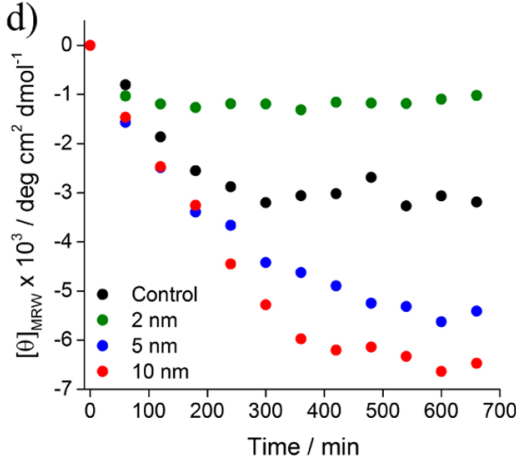

b)

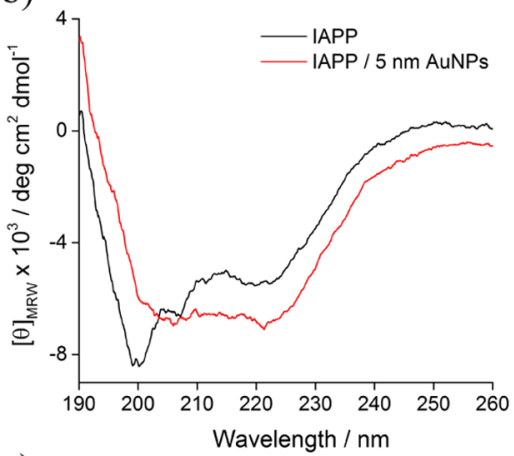

e)

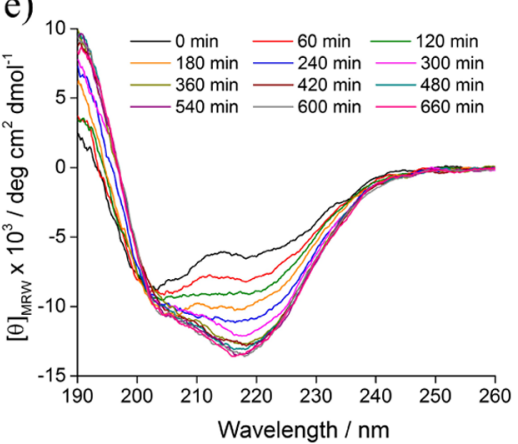

IAPP
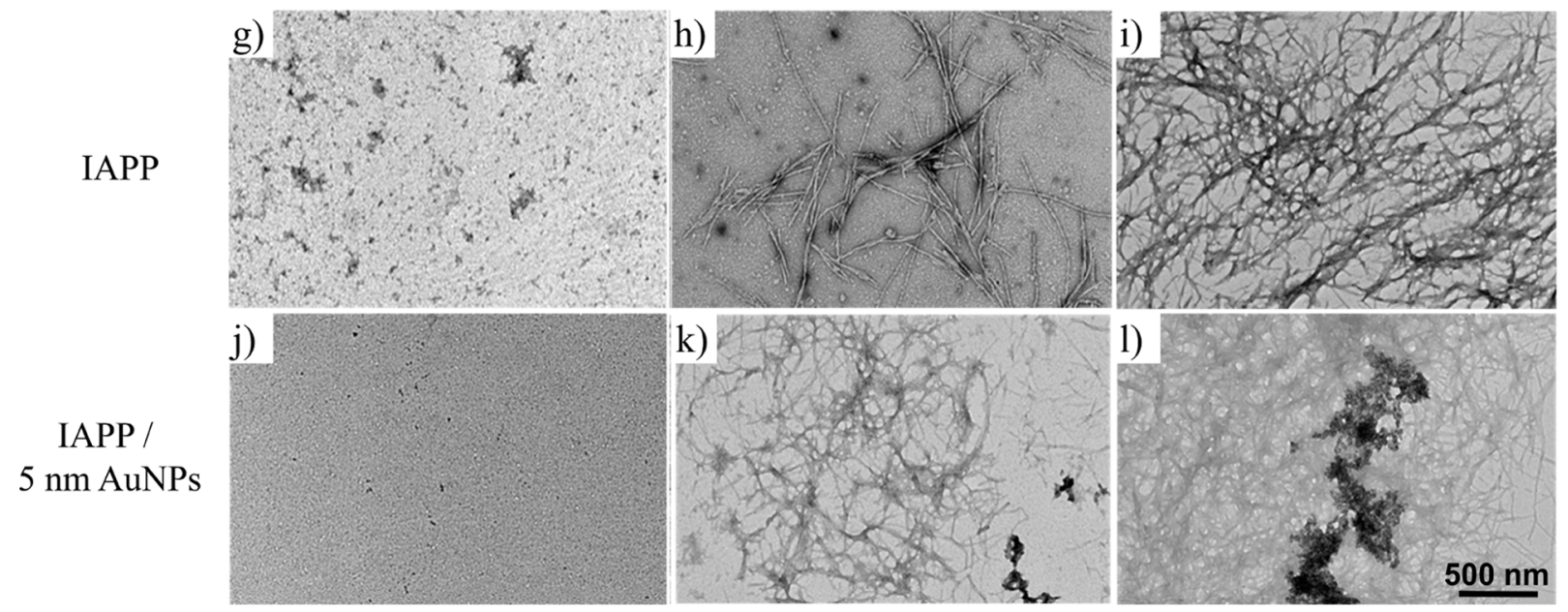

c)

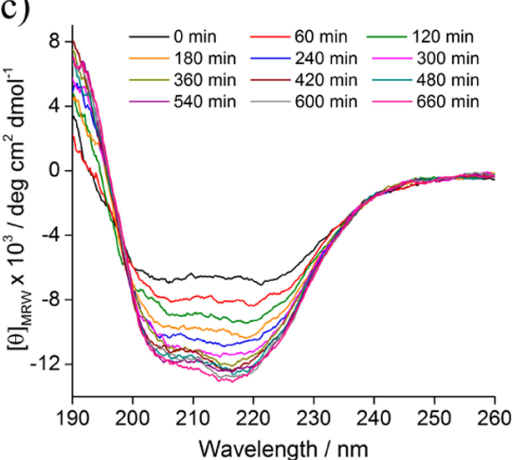

f)

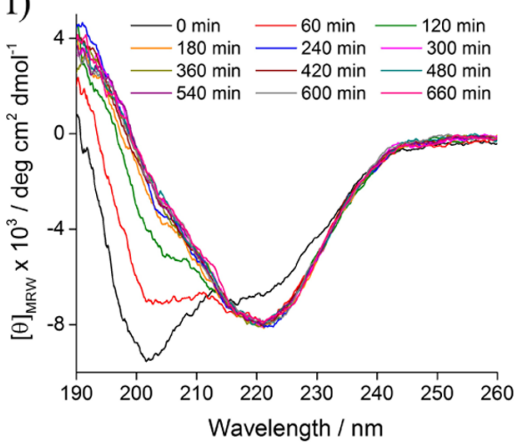

Figure 4. (a) IAPP fibrillation kinetics in solution recorded by CD. The arrows show a decreasing intensity of the signal at $200 \mathrm{~nm}$ and an increasing intensity of the signal at $220 \mathrm{~nm}$ as a function of time, representing the conformational change. (b) CD spectra of IAPP with and without $5 \mathrm{~nm}$ AuNPs $(t=0 \mathrm{~min})$, showing the rapid conformational change induced by AuNPs. (c) CD kinetics of IAPP $(25 \mu \mathrm{M})$ in the presence of $5 \mathrm{~nm}$ AuNPs $(20.8 \mathrm{nM})$. (d) CD intensity at $220 \mathrm{~nm}$, showing the accelerated formation of ordered structures (i.e., $\alpha$-helices and $\beta$-sheets) by 5 and $10 \mathrm{~nm}$ AuNPs but not smaller AuNPs $(2 \mathrm{~nm}$ ). The signals were subtracted from the values at time zero. (e and f) CD kinetics of IAPP (25 $\mu \mathrm{M})$ in the presence of 10 and $2 \mathrm{~nm}$ AuNPs, respectively. The final concentrations of the AuNPs were 2.08 and $20.8 \mathrm{nM}$, respectively. TEM images of IAPP solution in the absence $(\mathrm{g}-\mathrm{i})$ and presence $(\mathrm{j}-\mathrm{l})$ of $5 \mathrm{~nm}$ AuNPs $(20.8 \mathrm{nM})$ at different fibrillation time points $(0,4$, and $8 \mathrm{~h}$ from left to right, respectively).

weaker (water-mediated) binding of IAPP to the $\mathrm{Au}(100)$ surface, which was in agreement with other studies. ${ }^{64}$ The facetdependent structuring of water on Au surfaces had similar effects in both simulations for IAPP monomers and fibrils. Citrate molecules also exhibited stronger adsorption on the $\mathrm{Au}(100)$ facet than on the $\mathrm{Au}(111)$ facet (Figure S10), which resulted in a weaker IAPP-Au interaction in the former. Upon comparison of IAPP monomers to fibrils, it is worth noting that the small decrease in citrate density close to the $\mathrm{Au}(111)$ surface, caused by the displacement of citrate molecules upon binding, suggests a stronger binding of monomers rather than fibrils to the Au surface.
In summary, our results show that the IAPP-AuNP interaction is dominated by the $\mathrm{N}$-terminal domain of the peptide, with the conformation of surface-adsorbed IAPP determined by the exposed Au facets. Water/citrate-mediated binding on the $\mathrm{Au}(100)$ facet led to the stabilization of a helical conformation at the $\mathrm{N}$-terminal domain, with slight unfolding at the amyloidogenic helix, while a complete unfolding at the amyloidogenic region of IAPP on the $\mathrm{Au}(111)$ facet was observed because of direct binding to the Au surface. Interestingly, although both binding mechanisms showed the exposure of the amyloidogenic C-terminal domain of the IAPP monomers, the stronger deformation of the IAPP structure 

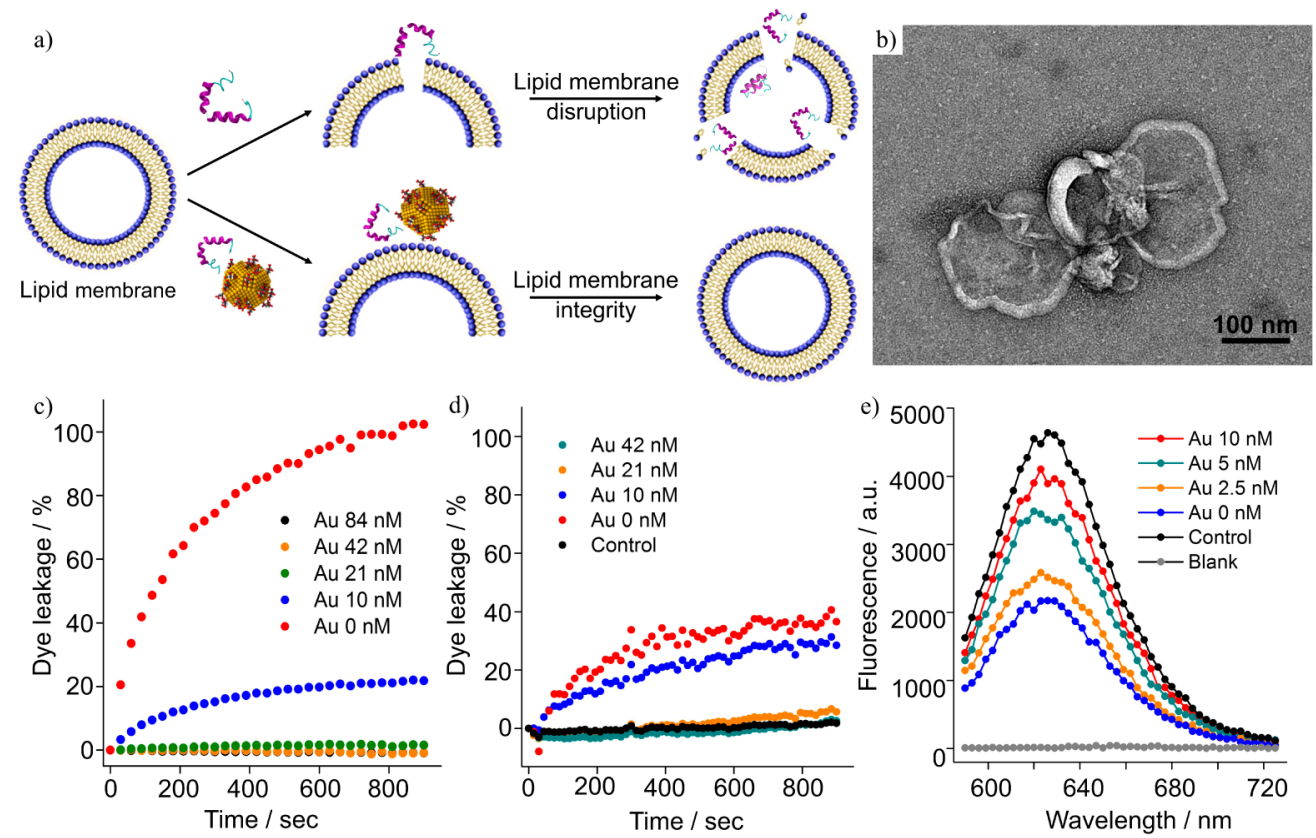

Figure 5. (a) Schematic of IAPP-induced lipid membrane disruption, which could be inhibited by AuNPs. (b) TEM image showing the interaction of IAPP with POPC/POPG (50:50) liposomes that disrupted the lipid membranes. (c and d) Time-dependent dye leakage from CF-encapsulated POPC/POPG (50:50) liposomes triggered by IAPP oligomers and fibrils, respectively, in the presence of AuNPs (5 nm) at different concentrations. The concentrations of IAPP oligomers and fibrils were 0.31 and $1 \mu \mathrm{M}$, respectively. Fluorescence was recorded with excitation at $485 \mathrm{~nm}$ and emission at $520 \mathrm{~nm} ; 100 \%$ CF release was obtained by adding Triton X-100 to the solution. (e) Fluorescence emission of NR (10 $\mu \mathrm{M})$ in the liposome (DMPC/DMPG, 50:50)/LAPP solution. The control sample contains liposomes and NR dye, and the blank sample is the NR only solution.

could suggest the $\mathrm{Au}(111)$ surface to be a stronger fibril promoter. Previous studies have demonstrated the increase in $(111) /(100)$ dominant facet ratios with an increase in AuNP size. ${ }^{65}$ Therefore, the IAPP adsorption and fibrillation kinetics could potentially be mediated by a change in the size and morphology of NPs.

Regulation of IAPP Fibrillation Kinetics by the Presence of AuNPs. Similar to other amyloid peptides, the amyloidogenesis of IAPP is a multistep process that is highly dependent on the formation of energetically unfavorable nuclei. In general, the nucleation-growth process of IAPP fibrillation is slow and requires a high concentration of monomers. This could be observed by the utilization of the widely used thioflavin $\mathrm{T}$ (ThT) assay, in which the fluorophore targets the formation of $\beta$-sheet structure (Figure S11a). Amyloid formation proceeded slowly before a sufficient population of nuclei were formed, during which only trace amounts of amyloid fibrils could be found. Once a critical concentration of nuclei was reached, peptide assembly proceeded rapidly for conversion into fibrils, evidenced by an exponential increase in ThT fluorescence. This indicated the formation of mature fibrils, which was observed by TEM to have an average width of $9.1 \pm 1.8 \mathrm{~nm}$ and a micrometer length (Figure S11b). ${ }^{8,66}$ The fibrillation kinetics were found to be strongly dependent on IAPP concentration, with a higher concentration of IAPP resulting in a shorter lag phase and higher ThT fluorescence.

The effect of AuNPs on IAPP amyloidogenesis was investigated by considering the strong affinity of IAPP for AuNPs, and the facet-dependent adsorption mechanisms suggested by the MD simulations. Circular dichroism (CD), which is commonly used to study the secondary structure of peptides and/or proteins, was used to monitor the IAPP conformational changes during the fibrillation process. In solution, IAPP transforms from random coil into $\beta$-sheet during the growth process, with typical CD signals at 200 and $220 \mathrm{~nm}$ being observed, respectively (Figure 4a).

Interestingly, we observed a conformational change from random coil $(200 \mathrm{~nm})$ into a helical (205 and $222 \mathrm{~nm}$ ) intermediate structure in the presence of $5 \mathrm{~nm}$ AuNPs (Figure $4 \mathrm{~b}, \mathrm{c}$ and Figure S12a), which were dominated by the (111) facets. ${ }^{45}$ We also demonstrated that this effect was not due to the absorbance of AuNPs or spectral overlap between AuNPs and IAPP (Figure S12b,c). In addition, the amounts of ordered structures (i.e., $\alpha$-helices and $\beta$-sheets) were found to increase in the presence of $5 \mathrm{~nm}$ AuNPs as the CD intensity at $220 \mathrm{~nm}$ was shown to be higher (Figure $4 \mathrm{~d}$ and Figure S13). This agrees with the TEM results that show extensive fibrillar bundles after $4 \mathrm{~h}$ in the presence of $5 \mathrm{~nm}$ AuNPs (Figure $4 \mathrm{~g}-1$ ). AuNP-promoted fibrillation was also demonstrated by TEM at shorter fibrillation periods [i.e., 10, 30, and $60 \mathrm{~min}$ (Figure S14)]. These results suggested that the presence of AuNPs could increase the level of ordering of amyloid structures and accelerate the fibrillation process, which could be rationalized by the fact that the local concentration of IAPP increases upon binding of IAPP to the NP surface. The increased effective concentration of IAPP at the surface of the AuNPs then favors nucleation and promotes fibrillation. This effect has been reported in the literature where liquid-solid interfaces exhibited specific and significant effects on promoting amyloid fibrillation by forming a critical nucleus for the nucleation of peptide fibrils at an interface. ${ }^{21,43}$ It is therefore expected that AuNPs with a larger surface area may exert a stronger influence on IAPP fibrillation. Indeed, $10 \mathrm{~nm}$ AuNPs have shown a capability to accelerate fibrillation at a much lower concentration $[\sim 2.08 \mathrm{nM}$ (Figure 4d,e, Figure S13, and Figure S15ac) similar to that of $5 \mathrm{~nm}$ AuNPs $(\sim 20.8 \mathrm{nM})$, supporting the 
conclusion of size-dependent IAPP-AuNP interactions. The effect of $2 \mathrm{~nm}$ AuNPs on IAPP fibrillation was not obvious, and some inhibitory effect was observed (Figure 4d,f, Figure S13, and Figure S15d-f). This was likely due to the smaller surface area and the lack of a dominating (111) facet. ${ }^{67}$

The rapid formation of helical intermediate structures on the $\mathrm{Au}$ surface could be another factor that promoted IAPP fibrillation. This hypothesis was also supported by MD simulation, which showed a relatively stable helical structure at the N-terminal domain for the adsorption of IAPP on Au surfaces (Figure 3). Recent studies have demonstrated that helical structures are on-pathway intermediates to the formation of intermolecular $\beta$-sheets and subsequent amyloid formation. ${ }^{9,68}$ In summary, we proposed that the strong binding of AuNPs enhanced the local population of peptides and favored the formation of an intermediate helix with exposed amyloidogenic regions on the Au surface, promoting IAPP selfassembly. NP-promoted amyloid fibrillation was also observed for short peptides (e.g., NNFGAIL from IAPP and GNNQQNY from prion proteins). ${ }^{69,70}$ In these two cases, although rapid oligomerization was triggered by the NP surfaces that were densely packed with $\mathrm{N}$-terminally bound peptides, the surfaces did not alter the initial peptide configurations.

Inhibition of IAPP-Induced Lipid Membrane Disruption by AuNPs. The loss of lipid membrane integrity has been considered as the major cytotoxic mechanism induced by IAPP. ${ }^{26,36}$ The molecular basis of the lipid-IAPP interaction is known to depend on the concentration and charge density of the lipid membrane, which can occur over a physiologically relevant range of anionic content. ${ }^{31}$ It is also known that IAPP residues 1-19 are responsible for the membrane disruption. $^{31,71}$ Because AuNPs have been shown to bind to the Nterminal domain of IAPP, we hypothesized that AuNPs could inhibit the association of IAPP with negatively charged lipid membranes, preventing membrane disruption (Figure 5a). To test this hypothesis, a liposome model composed of 1palmitoyl-2-oleoyl-sn-glycero-3-phosphocholine (POPC) and 1-palmitoyl-2-oleoyl-sn-glycero-3-phospho( $1^{\prime}$-rac-glycerol $)$ (POPG) with 50\% PG content was prepared by a typical hydration method and size exclusion, ${ }^{29}$ to give liposomes with an average size of $151 \pm 1.7 \mathrm{~nm}$ (Figure S16a,b) and a surface charge of $-59.3 \pm 4.0 \mathrm{mV}$. TEM imaging revealed the IAPPliposome interactions, in which the peptide can disrupt the membrane surface (Figure $5 \mathrm{~b}$ ) and penetrate through the lipid structure during growth into larger fibrils (Figure S17).

To quantify the membrane disruption by IAPP and the inhibitory effect of AuNPs, a dye leakage assay was performed. To this end, a high concentration $(50 \mathrm{mM})$ of carboxyfluorescein (CF), a self-quenching fluorescent dye, was encapsulated into the POPC/POPG liposomes. At such high concentrations of $\mathrm{CF}$, the emission was quenched, and the recovery of fluorescence was measured during the dye leakage induced by IAPP (Figure S18). Because both the AuNPs and lipid membranes are negatively charged, implying a passive process for the AuNP-lipid interaction, the dye leakage from the POPC/POPG liposomes was anticipated to be induced by the IAPP-lipid interactions. As shown in Figure $5 \mathrm{c}$, an immediate release of CF from the liposome was triggered by IAPP oligomers $(0.31 \mu \mathrm{M})$, whereas reduced and delayed dye leakage was observed in the presence of $5 \mathrm{~nm}$ AuNPs within the experimental time frame, indicating that lipid membrane disruption by IAPP could be inhibited by AuNPs. Similarly, lipid membrane leakage could also be induced by IAPP fibrils
(1 $\mu \mathrm{M}$ ) with much less CF release (40\%) within the experimental time frame (Figure 5d), which also could be inhibited by $5 \mathrm{~nm}$ AuNPs. It is noted that IAPP oligomers are more efficient in causing membrane leakage than IAPP fibrils, which is consistent with the findings that IAPP oligomers are responsible for the cytotoxicity. ${ }^{17,19,21,68}$ The inhibition efficiencies of AuNPs were concentration-dependent; i.e., higher AuNP concentrations led to an increased level of inhibition. Because the AuNPs (5 nm) consist of (111) facetdominated surfaces, facilitating significant conformational changes of IAPP and large binding sites for surface interactions (Figure $1 \mathrm{c}$ and Figure S9b), ${ }^{50,72}$ a strong inhibition of IAPPinduced lipid membrane disruption was achieved by AuNPs.

The lipid membrane micropolarity was also tracked to investigate the effect of IAPP-AuNPs interactions on peptideinduced membrane disruption. Here, an environmentally sensitive fluorescent dye Nile red (NR) was used, which fluoresces when bound to hydrophobic structures. It is reported that the fluorescence intensity of NR is substantially enhanced in hydrophobic environments compared to that in hydrophilic environments. $^{71}$ As shown in Figure 5e, the presence of DMPC/DMPG liposomes $[156.4 \pm 1.1 \mathrm{~nm},-70.5 \pm 4.5 \mathrm{mV}$ (Figure S16c,d)] significantly increases the fluorescence emission of NR, indicating the hydrophobic dye was embedded in the lipid bilayer of the liposome. A peak blue-shift of NR emission from 650 to $625 \mathrm{~nm}$ was also noted in the liposome solution compared to that in buffer, confirming the solubilization of NR by the lipid membrane. In the presence of IAPP, the fluorescence intensity of NR was reduced to $46 \%$, suggesting an increase in environment micropolarity. This is because the penetration of IAPP into the lipid membrane might lead to a looser packing of lipid molecules and the intrusion of water, both of which can increase the polarity of lipid membranes. In the presence of $5 \mathrm{~nm}$ AuNPs, the fluorescence was regained with an increasing AuNP concentration, implying the integrity of the lipid membrane was maintained in the presence of AuNPs.

\section{CONCLUSIONS}

Our combined experimental and computational simulation study has demonstrated a strong interaction between full-length IAPP and AuNPs, which is initiated by the hydrophilic Nterminal domain, followed by a conformational change in a facet-dependent manner. The binding of IAPP to AuNPs induces the aggregation of AuNPs, with a stronger aggregation being noted for larger NPs. Because IAPP-AuNP interactions were shown to originate from the outer hydrophilic N-terminal sequence rather than the amyloidogenic core sequence, the exposed N-terminal domain of preformed IAPP fibrils could induce adsorption and/or assembly of AuNPs or direct in situ $\mathrm{Au}$ nucleation on the fibril surface.

The IAPP-AuNP interaction was observed to accelerate IAPP fibrillation by the preferential formation of ordered structures, which can be partially ascribed to the AuNP-induced formation of an intermediate helix and conformational restructuring of the amyloidogenic region that consequently promoted IAPP fibrillation. This was supported by MD simulations showing significant conformational rearrangements on $\mathrm{Au}(111)$ facets that are dominant in larger AuNPs. In addition, the large surface area of AuNPs and high peptide adsorption affinity led to an increased local concentration of peptide, thus triggering nucleation and further fibril growth. By using synthetic liposome models, we have demonstrated the 
inhibition of IAPP-triggered lipid membrane disruption by implementing IAPP-AuNP interactions. We expect that investigation of the availability of amyloid peptide/AuNPbinding domains will provide a deeper understanding of the responsible structural features for NP-driven fibril formation. This in turn will provide fundamental insight and guidelines for controlling the interactions of biomolecules with NPs for amyloid diseases.

\section{ASSOCIATED CONTENT}

\section{S Supporting Information}

The Supporting Information is available free of charge on the ACS Publications website at DOI: 10.1021/acs.chemmater.6b04144.

Materials and methods, including IAPP preparation, DOSY-NMR, CPMG acquisition, ITC, gel electrophoresis, UV-vis spectroscopy, AFM imaging, TEM imaging, $\mathrm{MD}$ simulation, $\mathrm{CD}$, the ThT fluorescence assay, liposome preparation, DLS, and the dye leakage assay (PDF)

\section{AUTHOR INFORMATION}

\section{Corresponding Author}

*E-mail: m.stevens@imperial.ac.uk.

ORCID ${ }^{\oplus}$

Molly M. Stevens: 0000-0002-7335-266X

Notes

The authors declare no competing financial interest.

For raw data requests, please contact m.stevens@imperial.ac.uk.

\section{ACKNOWLEDGMENTS}

S.-T.W. is thankful for the financial support from the Taiwan Strategic Alliance for the Ph.D. study. I.Y. and M.M.S. acknowledge the Australian Research Council for financial support under the Discovery Project scheme (DP140101888). This research was undertaken with the assistance of computational resources from the National Computational Infrastructure (NCI) and Victorian Life Sciences Computation Initiative (VLSCI) Grants VR0028 and VR0202. This work was also supported by the Wellcome Trust (Senior Investigator Award 100280 and Multiuser NMR Experiment Grant 104833 to S.J.M.). M.M. was supported by Marie Curie actions FP7 through the Intra-European Marie Curie Fellowship "Time to Mature" under grant agreement no. 275005. S.R. was supported by Marie Curie actions FP7 through the Intra-European Marie Curie Fellow-ship "FunStar Capsule" under grant agreement no. 629218. N.A. was supported by Marie Curie actions FP7 through the Intra-European Marie Curie Fellowship "ConPilus" under grant agreement no. 623123 .

\section{REFERENCES}

(1) Kahn, S. E. The Relative Contributions of Insulin Resistance and Beta-Cell Dysfunction to the Pathophysiology of Type 2 Diabetes. Diabetologia 2003, 46, 3-19.

(2) Epstein, F. H.; Höppener, J. W. M.; Ahrén, B.; Lips, C. J. M. Islet Amyloid and Type 2 Diabetes Mellitus. N. Engl. J. Med. 2000, 343, 411-419.

(3) Cooper, G. J.; Willis, A. C.; Clark, A.; Turner, R. C.; Sim, R. B.; Reid, K. B. Purification and Characterization of a Peptide from Amyloid-Rich Pancreases of Type 2 Diabetic Patients. Proc. Natl. Acad. Sci. U. S. A. 1987, 84, 8628-8632.
(4) Butler, A. E.; Janson, J.; Bonner-Weir, S.; Ritzel, R.; Rizza, R. A.; Butler, P. C. B-Cell Deficit and Increased B-Cell Apoptosis in Humans with Type 2 Diabetes. Diabetes 2003, 52, 102-110.

(5) Clark, A.; Lewis, C. E.; Willis, A. C.; Cooper, G. J. S.; Morris, J. F.; Reid, K. B. M.; Turner, R. C. Islet Amyloid Formed from DiabetesAssociated Peptide May Be Pathogenic in Type-2 Diabetes. Lancet 1987, 330, 231-234.

(6) Jaikaran, E. T.; Clark, A. Islet Amyloid and Type 2 Diabetes: From Molecular Misfolding to Islet Pathophysiology. Biochim. Biophys. Acta, Mol. Basis Dis. 2001, 1537, 179-203.

(7) Wang, C.; Yang, A.; Li, X.; Li, D.; Zhang, M.; Du, H.; Li, C.; Guo, Y.; Mao, X.; Dong, M.; et al. Observation of Molecular Inhibition and Binding Structures of Amyloid Peptides. Nanoscale 2012, 4, 18951909.

(8) Westermark, P.; Engström, U.; Johnson, K. H.; Westermark, G. T.; Betsholtz, C. Islet Amyloid Polypeptide: Pinpointing Amino Acid Residues Linked to Amyloid Fibril Formation. Proc. Natl. Acad. Sci. U. S. A. 1990, 87, 5036-5040.

(9) Kayed, R.; Head, E.; Thompson, J. L.; McIntire, T. M.; Milton, S. C.; Cotman, C. W.; Glabe, C. G. Common Structure of Soluble Amyloid Oligomers Implies Common Mechanism of Pathogenesis. Science 2003, 300, 486-489.

(10) Westermark, P.; Li, Z. C.; Westermark, G. T.; Leckstrom, A.; Steiner, D. F. Effects of Beta Cell Granule Components on Human Islet Amyloid Polypeptide Fibril Formation. FEBS Lett. 1996, 379, 203-206.

(11) Peacock, H.; Luo, J.; Yamashita, T.; Luccarelli, J.; Thompson, S.; Hamilton, A. D. Non-Covalent S...O Interactions Control Conformation in a Scaffold That Disrupts Islet Amyloid Polypeptide Fibrillation. Chem. Sci. 2016, 7, 6435-6439.

(12) Kumar, S.; Birol, M.; Schlamadinger, D. E.; Wojcik, S. P.; Rhoades, E.; Miranker, A. D. Foldamer-Mediated Manipulation of a Pre-Amyloid Toxin. Nat. Commun. 2016, 7, 11412.

(13) Susa, A. C.; Wu, C.; Bernstein, S. L.; Dupuis, N. F.; Wang, H.; Raleigh, D. P.; Shea, J.-E.; Bowers, M. T. Defining the Molecular Basis of Amyloid Inhibitors: Human Islet Amyloid Polypeptide-Insulin Interactions. J. Am. Chem. Soc. 2014, 136, 12912-12919.

(14) Yan, L. M.; Velkova, A.; Tatarek-Nossol, M.; Andreetto, E.; Kapurniotu, A. Iapp Mimic Blocks Abeta Cytotoxic Self-Assembly: Cross-Suppression of Amyloid Toxicity of Abeta and Iapp Suggests a Molecular Link between Alzheimer's Disease and Type Ii Diabetes. Angew. Chem., Int. Ed. 2007, 46, 1246-1252.

(15) Middleton, C. T.; Marek, P.; Cao, P.; Chiu, C.-c.; Singh, S.; Woys, A. M.; de Pablo, J. J.; Raleigh, D. P.; Zanni, M. T. TwoDimensional Infrared Spectroscopy Reveals the Complex Behaviour of an Amyloid Fibril Inhibitor. Nat. Chem. 2012, 4, 355-360.

(16) Song, Y.; Cheng, P.-N.; Zhu, L.; Moore, E. G.; Moore, J. S. Multivalent Macromolecules Redirect Nucleation-Dependent Fibrillar Assembly into Discrete Nanostructures. J. Am. Chem. Soc. 2014, 136, 5233-5236.

(17) Engel, M. F.; Khemtemourian, L.; Kleijer, C. C.; Meeldijk, H. J.; Jacobs, J.; Verkleij, A. J.; de Kruijff, B.; Killian, J. A.; Hoppener, J. W. Membrane Damage by Human Islet Amyloid Polypeptide through Fibril Growth at the Membrane. Proc. Natl. Acad. Sci. U. S. A. 2008, 105, 6033-6038.

(18) Glabe, C. G. Common Mechanisms of Amyloid Oligomer Pathogenesis in Degenerative Disease. Neurobiol. Aging 2006, 27, $570-575$.

(19) Haataja, L.; Gurlo, T.; Huang, C. J.; Butler, P. C. Islet Amyloid in Type 2 Diabetes, and the Toxic Oligomer Hypothesis. Endocr. Rev. 2008, 29, 303-316.

(20) Kayed, R.; Sokolov, Y.; Edmonds, B.; McIntire, T. M.; Milton, S. C.; Hall, J. E.; Glabe, C. G. Permeabilization of Lipid Bilayers Is a Common Conformation-Dependent Activity of Soluble Amyloid Oligomers in Protein Misfolding Diseases. J. Biol. Chem. 2004, 279, 46363-46366.

(21) Last, N. B.; Rhoades, E.; Miranker, A. D. Islet Amyloid Polypeptide Demonstrates a Persistent Capacity to Disrupt Membrane Integrity. Proc. Natl. Acad. Sci. U. S. A. 2011, 108, 9460-9465. 
(22) Monsellier, E.; Bousset, L.; Melki, R. A-Synuclein and Huntingtin Exon 1 Amyloid Fibrils Bind Laterally to the Cellular Membrane. Sci. Rep. 2016, 6, 19180.

(23) Xue, W.-F.; Hellewell, A. L.; Gosal, W. S.; Homans, S. W.; Hewitt, E. W.; Radford, S. E. Fibril Fragmentation Enhances Amyloid Cytotoxicity. J. Biol. Chem. 2009, 284, 34272-34282.

(24) Meyer-Luehmann, M.; Spires-Jones, T. L.; Prada, C.; GarciaAlloza, M.; de Calignon, A.; Rozkalne, A.; Koenigsknecht-Talboo, J.; Holtzman, D. M.; Bacskai, B. J.; Hyman, B. T. Rapid Appearance and Local Toxicity of Amyloid-[Bgr] Plaques in a Mouse Model of Alzheimer/'S Disease. Nature 2008, 451, 720-724.

(25) Paravastu, A. K.; Qahwash, I.; Leapman, R. D.; Meredith, S. C.; Tycko, R. Seeded Growth of B-Amyloid Fibrils from Alzheimer's Brain-Derived Fibrils Produces a Distinct Fibril Structure. Proc. Natl. Acad. Sci. U. S. A. 2009, 106, 7443-7448.

(26) Mirzabekov, T. A.; Lin, M.-c.; Kagan, B. L. Pore Formation by the Cytotoxic Islet Amyloid Peptide Amylin. J. Biol. Chem. 1996, 271, 1988-1992.

(27) Quist, A.; Doudevski, I.; Lin, H.; Azimova, R.; Ng, D.; Frangione, B.; Kagan, B.; Ghiso, J.; Lal, R. Amyloid Ion Channels: A Common Structural Link for Protein-Misfolding Disease. Proc. Natl. Acad. Sci. U. S. A. 2005, 102, 10427-10432.

(28) Bram, Y.; Frydman-Marom, A.; Yanai, I.; Gilead, S.; ShaltielKaryo, R.; Amdursky, N.; Gazit, E. Apoptosis Induced by Islet Amyloid Polypeptide Soluble Oligomers Is Neutralized by Diabetes-Associated Specific Antibodies. Sci. Rep. 2014, 4, 4267.

(29) Lopes, D. H. J.; Meister, A.; Gohlke, A.; Hauser, A.; Blume, A.; Winter, R. Mechanism of Islet Amyloid Polypeptide Fibrillation at Lipid Interfaces Studied by Infrared Reflection Absorption Spectroscopy. Biophys. J. 2007, 93, 3132-3141.

(30) Jayasinghe, S. A.; Langen, R. Lipid Membranes Modulate the Structure of Islet Amyloid Polypeptide. Biochemistry 2005, 44, 1211312119 .

(31) Knight, J. D.; Miranker, A. D. Phospholipid Catalysis of Diabetic Amyloid Assembly. J. Mol. Biol. 2004, 341, 1175-1187.

(32) Farese, R. V.; Dimarco, P. E.; Barnes, D. E.; Sabir, M. A.; Larson, R. E.; Davis, J. S.; Morrison, A. D. Rapid Glucose-Dependent Increases in Phosphatidic Acid and Phosphoinositides in Rat Pancreatic Islets. Endocrinology 1986, 118, 1498-1503.

(33) Brender, J. R.; Lee, E. L.; Cavitt, M. A.; Gafni, A.; Steel, D. G.; Ramamoorthy, A. Amyloid Fiber Formation and Membrane Disruption Are Separate Processes Localized in Two Distinct Regions of Iapp, the Type-2-Diabetes-Related Peptide. J. Am. Chem. Soc. 2008, 130, 6424-6429.

(34) Nanga, R. P. R.; Brender, J. R.; Xu, J.; Hartman, K.; Subramanian, V.; Ramamoorthy, A. Three-Dimensional Structure and Orientation of Rat Islet Amyloid Polypeptide Protein in a Membrane Environment by Solution Nmr Spectroscopy. J. Am. Chem. Soc. 2009, 131, 8252-8261.

(35) Williamson, J. A.; Loria, J. P.; Miranker, A. D. Helix Stabilization Precedes Aqueous and Bilayer-Catalyzed Fiber Formation in Islet Amyloid Polypeptide. J. Mol. Biol. 2009, 393, 383-396.

(36) Cao, P.; Abedini, A.; Wang, H.; Tu, L. H.; Zhang, X.; Schmidt, A. M.; Raleigh, D. P. Islet Amyloid Polypeptide Toxicity and Membrane Interactions. Proc. Natl. Acad. Sci. U. S. A. 2013, 110, 19279-19284.

(37) Nel, A. E.; Madler, L.; Velegol, D.; Xia, T.; Hoek, E. M. V.; Somasundaran, P.; Klaessig, F.; Castranova, V.; Thompson, M. Understanding Biophysicochemical Interactions at the Nano-Bio Interface. Nat. Mater. 2009, 8, 543-557.

(38) Katz, E.; Willner, I. Integrated Nanoparticle-Biomolecule Hybrid Systems: Synthesis, Properties, and Applications. Angew. Chem., Int. Ed. 2004, 43, 6042-6108.

(39) Lundqvist, M.; Stigler, J.; Elia, G.; Lynch, I.; Cedervall, T.; Dawson, K. A. Nanoparticle Size and Surface Properties Determine the Protein Corona with Possible Implications for Biological Impacts. Proc. Natl. Acad. Sci. U. S. A. 2008, 105, 14265-14270.

(40) Cedervall, T.; Lynch, I.; Lindman, S.; Berggard, T.; Thulin, E.; Nilsson, H.; Dawson, K. A.; Linse, S. Understanding the Nanoparticle-
Protein Corona Using Methods to Quantify Exchange Rates and Affinities of Proteins for Nanoparticles. Proc. Natl. Acad. Sci. U. S. A. 2007, 104, 2050-2055.

(41) Gebauer, J. S.; Malissek, M.; Simon, S.; Knauer, S. K.; Maskos, M.; Stauber, R. H.; Peukert, W.; Treuel, L. Impact of the Nanoparticle-Protein Corona on Colloidal Stability and Protein Structure. Langmuir 2012, 28, 9673-9679.

(42) Shemetov, A. A.; Nabiev, I.; Sukhanova, A. Molecular Interaction of Proteins and Peptides with Nanoparticles. ACS Nano 2012, 6, 4585-4602.

(43) Linse, S.; Cabaleiro-Lago, C.; Xue, W. F.; Lynch, I.; Lindman, S.; Thulin, E.; Radford, S. E.; Dawson, K. A. Nucleation of Protein Fibrillation by Nanoparticles. Proc. Natl. Acad. Sci. U. S. A. 2007, 104, $8691-8696$

(44) Todorova, N.; Makarucha, A. J.; Hine, N. D.; Mostofi, A. A.; Yarovsky, I. Dimensionality of Carbon Nanomaterials Determines the Binding and Dynamics of Amyloidogenic Peptides: Multiscale Theoretical Simulations. PLoS Comput. Biol. 2013, 9, e1003360.

(45) Yoo, S. I.; Yang, M.; Brender, J. R.; Subramanian, V.; Sun, K.; Joo, N. E.; Jeong, S.-H.; Ramamoorthy, A.; Kotov, N. A. Inhibition of Amyloid Peptide Fibrillation by Inorganic Nanoparticles: Functional Similarities with Proteins. Angew. Chem., Int. Ed. 2011, 50, 5110-5115.

(46) Zhang, M.; Mao, X.; Yu, Y.; Wang, C.-X.; Yang, Y.-L.; Wang, C. Nanomaterials for Reducing Amyloid Cytotoxicity. Adv. Mater. 2013, $25,3780-3801$

(47) Cabaleiro-Lago, C.; Szczepankiewicz, O.; Linse, S. The Effect of Nanoparticles on Amyloid Aggregation Depends on the Protein Stability and Intrinsic Aggregation Rate. Langmuir 2012, 28, 18521857.

(48) Kim, Y.; Park, J.-H.; Lee, H.; Nam, J.-M. How Do the Size, Charge and Shape of Nanoparticles Affect Amyloid B Aggregation on Brain Lipid Bilayer? Sci. Rep. 2016, 6, 19548.

(49) Cabaleiro-Lago, C.; Quinlan-Pluck, F.; Lynch, I.; Dawson, K. A.; Linse, S. Dual Effect of Amino Modified Polystyrene Nanoparticles on Amyloid B Protein Fibrillation. ACS Chem. Neurosci. 2010, 1, 279287.

(50) Ali, S.; Myasnichenko, V. S.; Neyts, E. C. Size-Dependent Strain and Surface Energies of Gold Nanoclusters. Phys. Chem. Chem. Phys. 2016, 18, 792-800.

(51) Brancolini, G.; Corazza, A.; Vuano, M.; Fogolari, F.; Mimmi, M. C.; Bellotti, V.; Stoppini, M.; Corni, S.; Esposito, G. Probing the Influence of Citrate-Capped Gold Nanoparticles on an Amyloidogenic Protein. ACS Nano 2015, 9, 2600-2613.

(52) Álvarez, Y. D.; Fauerbach, J. A.; Pellegrotti, J. V.; Jovin, T. M.; Jares-Erijman, E. A.; Stefani, F. D. Influence of Gold Nanoparticles on the Kinetics of A-Synuclein Aggregation. Nano Lett. 2013, 13, 61566163.

(53) Shaw, C. P.; Middleton, D. A.; Volk, M.; Lévy, R. AmyloidDerived Peptide Forms Self-Assembled Monolayers on Gold Nanoparticle with a Curvature-Dependent B-Sheet Structure. ACS Nano 2012, 6, 1416-1426.

(54) Joshi, H.; Shirude, P. S.; Bansal, V.; Ganesh, K. N.; Sastry, M. Isothermal Titration Calorimetry Studies on the Binding of Amino Acids to Gold Nanoparticles. J. Phys. Chem. B 2004, 108, 1153511540.

(55) Zhong, Z.; Patskovskyy, S.; Bouvrette, P.; Luong, J. H. T.; Gedanken, A. The Surface Chemistry of Au Colloids and Their Interactions with Functional Amino Acids. J. Phys. Chem. B 2004, 108, 4046-4052.

(56) Johnson, C. S., Jr. Diffusion Ordered Nucelar Magnetic Resonance Spectroscopy Principles and Applications. Prog. Nucl. Magn. Reson. Spectrosc. 1999, 34, 203-256.

(57) Brender, J. R.; Krishnamoorthy, J.; Messina, G. M. L.; Deb, A.; Vivekanandan, S.; La Rosa, C.; Penner-Hahn, J. E.; Ramamoorthy, A. Zinc Stabilization of Prefibrillar Oligomers of Human Islet Amyloid Polypeptide. Chem. Commun. 2013, 49, 3339-3341.

(58) Salamekh, S.; Brender, J. R.; Hyung, S.-J.; Nanga, R. P. R.; Vivekanandan, S.; Ruotolo, B. T.; Ramamoorthy, A. A Two-Site 
Mechanism for the Inhibition of Iapp Amyloidogenesis by Zinc. J. Mol. Biol. 2011, 410, 294-306.

(59) Verwey, E. J. W.; Overbeek, J. T. G. Theory of the Stability of Lyophobic Colloids; Courier Corp.: Chelmsford, MA, 1999.

(60) You, C. C.; Agasti, S. S.; Rotello, V. M. Isomeric Control of Protein Recognition with Amino Acid- and Dipeptide-Functionalized Gold Nanoparticles. Chem. - Eur. J. 2008, 14, 143-150.

(61) Brewer, S. H.; Glomm, W. R.; Johnson, M. C.; Knag, M. K.; Franzen, S. Probing Bsa Binding to Citrate-Coated Gold Nanoparticles and Surfaces. Langmuir 2005, 21, 9303-9307.

(62) Kajava, A. V.; Aebi, U.; Steven, A. C. The Parallel Superpleated Beta-Structure as a Model for Amyloid Fibrils of Human Amylin. J. Mol. Biol. 2005, 348, 247-252.

(63) Kelly, K. L.; Coronado, E.; Zhao, L. L.; Schatz, G. C. The Optical Properties of Metal Nanoparticles: The Influence of Size, Shape, and Dielectric Environment. J. Phys. Chem. B 2003, 107, 668677.

(64) Wright, L. B.; Palafox-Hernandez, J. P.; Rodger, P. M.; Corni, S.; Walsh, T. R. Facet Selectivity in Gold Binding Peptides: Exploiting Interfacial Water Structure. Chem. Sci. 2015, 6, 5204-5214.

(65) Barnard, A. S.; Chen, Y. Kinetic Modelling of the ShapeDependent Evolution of Faceted Gold Nanoparticles. J. Mater. Chem. 2011, 21, 12239-12245.

(66) Westermark, P.; Andersson, A.; Westermark, G. T. Islet Amyloid Polypeptide, Islet Amyloid, and Diabetes Mellitus. Physiol. Rev. 2011, 91, 795-826.

(67) Charchar, P.; Christofferson, A. J.; Todorova, N.; Yarovsky, I. Understanding and Designing the Gold-Bio Interface: Insights from Simulations. Small 2016, 12, 2395-2418.

(68) Meier, J. J.; Kayed, R.; Lin, C.-Y.; Gurlo, T.; Haataja, L.; Jayasinghe, S.; Langen, R.; Glabe, C. G.; Butler, P. C. Inhibition of Human Iapp Fibril Formation Does Not Prevent B-Cell Death: Evidence for Distinct Actions of Oligomers and Fibrils of Human Iapp. Am. J. Physiol. Endocrinol. Metab. 2006, 291, E1317-E1324.

(69) Gladytz, A.; Abel, B.; Risselada, H. J. Gold-Induced Fibril Growth: The Mechanism of Surface-Facilitated Amyloid Aggregation. Angew. Chem., Int. Ed. 2016, 55, 11242-11246.

(70) Gladytz, A.; Wagner, M.; Häupl, T.; Elsner, C.; Abel, B. Structure-Making Effects of Metal Nanoparticles in Amyloid Peptide Fibrillation. Part. Part. Syst. Charact. 2015, 32, 573-582.

(71) Greenspan, P.; Mayer, E. P.; Fowler, S. D. Nile Red: A Selective Fluorescent Stain for Intracellular Lipid Droplets. J. Cell Biol. 1985, 100, 965-973.

(72) Grzelczak, M.; Perez-Juste, J.; Mulvaney, P.; Liz-Marzan, L. M. Shape Control in Gold Nanoparticle Synthesis. Chem. Soc. Rev. 2008, 37, 1783-1791. 